Supporting Information:

\title{
Enhancing Hydrodeoxygenation of Bio-oil via Bimetallic Ni- V Catalysts Modified by Cross-surface Migrated-carbon from Biochar
}

\author{
Yujian Wu, Yan Sun, Kaili Liang, Zhengguang Yang, Ren Tu, Xudong Fan, Shuchao Cheng, \\ Haipeng Yu, Enchen Jiang*, Xiwei Xu* \\ College of Materials and Energy, South China Agricultural University, Guangzhou \\ 510640, China
}

Corresponding Authors

(Xiwei Xu) E-mail: xuxiwei200801@hotmail.com

(Enchen Jiang) E-mail: jiangenchen200801@hotmail.com 


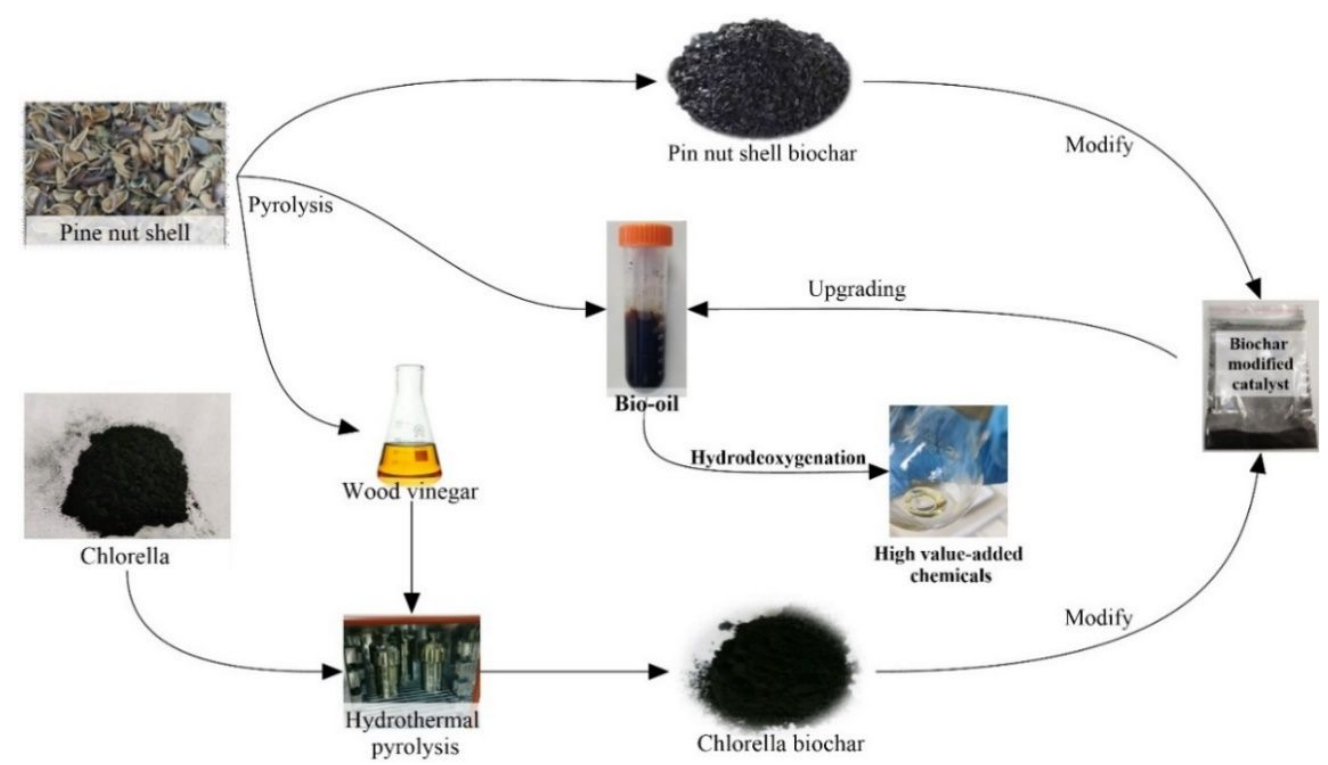

Scheme S1. A potential avenue for the HDO catalyst modification with biochar

Table S1. Carbon content of the fresh and spent catalysts

\begin{tabular}{|c|c|c|c|}
\hline Samples & $\mathrm{C}(\mathrm{wt} . \%)$ in fresh catalyst ${ }^{\mathrm{a}}$ & $\mathrm{C}(\mathrm{wt} . \%)$ in spent catalyst ${ }^{\mathrm{a}}$ & Carbon deposit ${ }^{\mathrm{b}}(\mathrm{wt} . \%)$ \\
\hline $\mathrm{H} \beta / \mathrm{Ni}-\mathrm{V}$ & 0.03 & 4.00 & 3.97 \\
\hline $\mathrm{PB} / \mathrm{Ni}-\mathrm{V}$ & 53.41 & 54.43 & 1.02 \\
\hline PB-H $\beta-4 / N i-V$ & 4.99 & 8.69 & 3.70 \\
\hline PB-H $\beta-8 / \mathrm{Ni}-\mathrm{V}$ & 1.61 & 4.94 & 3.33 \\
\hline PB-H $\beta-16 / \mathrm{Ni}-\mathrm{V}$ & 0.53 & 3.76 & 3.23 \\
\hline $\mathrm{H} \beta / \mathrm{Ni}-\mathrm{V}+\mathrm{PB} / \mathrm{Ni}-\mathrm{V}$ & 5.42 & 9.33 & 3.91 \\
\hline RB-H $\beta-8 / \mathrm{Ni}-\mathrm{V}$ & 1.29 & 5.16 & 3.87 \\
\hline EB-H $\beta-8 / \mathrm{Ni}-\mathrm{V}$ & 1.75 & 5.27 & 3.52 \\
\hline $\mathrm{CB}-\mathrm{H} \beta-8 / \mathrm{Ni}-\mathrm{V}$ & 3.17 & 5.94 & 2.77 \\
\hline AC-H $\beta-8 / \mathrm{Ni}-\mathrm{V}$ & 1.38 & 5.35 & 3.97 \\
\hline $\mathrm{AC} / \mathrm{Ni}-\mathrm{V}$ & 30.82 & 32.83 & 2.01 \\
\hline PB-H $\beta-8 / \mathrm{Ni}-\mathrm{V}-320^{\circ} \mathrm{C}$ & 1.61 & 6.45 & 4.84 \\
\hline $\mathrm{PB}-\mathrm{H} \beta-8 / \mathrm{Ni}-\mathrm{V}-380^{\circ} \mathrm{C}$ & 1.61 & 4.10 & 2.49 \\
\hline $\mathrm{PB}-\mathrm{H} \beta-8 / \mathrm{Ni}-\mathrm{V}-410^{\circ} \mathrm{C}$ & 1.61 & 3.12 & 1.51 \\
\hline PB-H $\beta-8 / \mathrm{Ni}-\mathrm{V}-\mathrm{WHSV}=0.3$ & 1.61 & 3.43 & 1.82 \\
\hline PB-H $\beta-8 / \mathrm{Ni}-\mathrm{V}-\mathrm{WHSV}=1$ & 1.61 & 5.21 & 3.60 \\
\hline PB-H $\beta-8 / \mathrm{Ni}-\mathrm{V}-\mathrm{WHSV}=2$ & 1.61 & 6.42 & 4.81 \\
\hline PB-H $\beta-8 / \mathrm{Ni}-\mathrm{V}_{\text {after reactivated 3-runs }}$ & 1.61 & 3.96 & 2.35 \\
\hline
\end{tabular}

a. Determined by Elemental analysis. 
b. Calculated by the difference between the carbon content of fresh catalyst and spent catalyst

Table S2. Characteristics of $\mathrm{H \beta}$ zeolite and the biochars

\begin{tabular}{|c|c|c|c|c|c|c|}
\hline & $\mathrm{H} \beta$ zeolite & $\mathrm{PB}$ & $\mathrm{RB}$ & EB & $\mathrm{CB}$ & $\mathrm{AC}$ \\
\hline \multicolumn{7}{|l|}{ Textural parameters ${ }^{\mathrm{a}}$} \\
\hline $\mathrm{S}_{\mathrm{BET}, \text { micro }}\left(\mathrm{m}^{2} \cdot \mathrm{g}^{-1}\right)$ & 420.63 & 0.65 & 0 & 0 & 0 & 201.4 \\
\hline $\mathrm{S}_{\mathrm{BET}, \text { external }}\left(\mathrm{m}^{2} \cdot \mathrm{g}^{-1}\right)$ & 157.76 & 1.53 & 4.88 & 3.15 & 1.96 & 201.8 \\
\hline $\mathrm{V}_{\mathrm{p}, \text { micro }}\left(\mathrm{cm}^{3} \cdot \mathrm{g}^{-1}\right)$ & 0.175 & 0.0003 & 0 & 0 & 0 & 0.082 \\
\hline$V_{p, \text { meso }}\left(\mathrm{cm}^{3} \cdot \mathrm{g}^{-1}\right)$ & 0.244 & 0.0024 & 0.0059 & 0.038 & 0.0006 & 0.263 \\
\hline $\mathrm{D}_{\text {pore }}(\mathrm{nm})$ & 9.1 & 11.3 & 9.6 & 5.4 & 2.5 & 5.4 \\
\hline $\begin{array}{l}\text { Elemental } \\
\text { composition (wt.\%) }\end{array}$ & N. d. ${ }^{c}$ & & & & & \\
\hline $\mathrm{C}$ & & 84.10 & 47.83 & 79.23 & 64.22 & 48.34 \\
\hline $\mathrm{H}$ & & 3.22 & 2.02 & 3.31 & 4.98 & 1.09 \\
\hline $\mathrm{N}$ & & 0.33 & 0.50 & 0.40 & 3.18 & 0.18 \\
\hline S & & 0.15 & 0.27 & 0.20 & 0.41 & 0.81 \\
\hline $\begin{array}{l}\text { Proximate analysis } \\
\text { (wt.\%) }\end{array}$ & N. d. ${ }^{c}$ & & & & & \\
\hline Moisture & & 3.2 & 8.8 & 8.3 & 5.3 & 9.6 \\
\hline Volatile & & 26 & 19.4 & 29.5 & 25.4 & 27.1 \\
\hline Ash & & 1.5 & 19.5 & 2.3 & 1.9 & 21.3 \\
\hline Fixed carbon ${ }^{b}$ & & 69.3 & 52.2 & 60.0 & 67.4 & 42.0 \\
\hline
\end{tabular}

a. Determined by $\mathrm{N}_{2}$ isotherm adsorption-desorption.

b. Determined by difference.

c. Not detected.

Table S3. TG-H ${ }_{2}$-TPR analysis of the $\mathrm{H \beta}$ zeolite, PB and selected catalysts.

\begin{tabular}{cccc}
\hline Samples & $\begin{array}{c}\text { Mass loss between } 50 \text { to } \\
185^{\circ} \mathrm{C}(\text { wt. } \%)\end{array}$ & $\begin{array}{c}\text { Mass loss between } 185 \text { to } \\
330{ }^{\circ} \mathrm{C}(\text { wt.\%) }\end{array}$ & $\begin{array}{c}\text { Mass loss between } 330 \text { to } \\
800{ }^{\circ} \mathrm{C} \text { (wt.\%) }\end{array}$ \\
\hline $\mathrm{H} \beta$ zeolite & 6.49 & 1.13 & 2.21 \\
$\mathrm{~PB}$ & 1.53 & 1.92 & 15.24 \\
$\mathrm{H} \beta / \mathrm{Ni}-\mathrm{V}$ & 14.29 & 20.87 & 6.74 \\
$\mathrm{~PB} / \mathrm{Ni}-\mathrm{V}$ & 3.73 & 14.67 & 23.03 \\
$\mathrm{~PB}-\mathrm{H} \beta-4 / \mathrm{Ni}-\mathrm{V}$ & 8.26 & 13.29 & 12.32 \\
$\mathrm{~PB}-\mathrm{H} \beta-8 / \mathrm{Ni}-\mathrm{V}$ & 11.04 & 16.56 & 10.48
\end{tabular}


Table S4. TG-TPO analysis of the PB modified Hß/Ni-V catalysts

\begin{tabular}{|c|c|c|c|c|}
\hline \multirow{3}{*}{ Samples } & \multicolumn{4}{|c|}{ TPO peaks temperature $\left({ }^{\circ} \mathrm{C}\right)$ of the fresh catalysts } \\
\hline & \multirow[b]{2}{*}{ Peak 1} & \multirow[b]{2}{*}{ Peak 2} & Peak 3 & Peak 4 \\
\hline & & & (residue-carbon) & (migrated-carbon) \\
\hline $\mathrm{H} \beta / \mathrm{Ni}-\mathrm{V}$ & 95 & 377 & - & - \\
\hline $\mathrm{PB} / \mathrm{Ni}-\mathrm{V}$ & 95 & 297 & 471 & - \\
\hline PB-Hß-4/Ni-V & 99 & 369 & $485(16.7 \%)$ & $541(83.3 \%)$ \\
\hline PB-H $\beta-8 / N i-V$ & 96 & 369 & $488(11.0 \%)$ & $550(89.0 \%)$ \\
\hline PB-Hß-16/Ni-V & 96 & 361 & - & $537(100 \%)$ \\
\hline $\mathrm{H} \beta / \mathrm{Ni}-\mathrm{V}+\mathrm{PB} / \mathrm{Ni}-\mathrm{V}$ & 95 & 364 & 478 & - \\
\hline
\end{tabular}

Table S5. Binding energy of $\mathrm{C} 1 \mathrm{~s}$, Si $2 \mathrm{p}$ and corresponding surface concentration of the selected catalysts.

\begin{tabular}{cccc}
\hline Samples & $\mathrm{C}-\mathrm{O}$ & $\mathrm{C}=\mathrm{O}$ & $\mathrm{SiO}_{2}$ \\
$\mathrm{H} \beta / \mathrm{Ni}-\mathrm{V}$ & - & 289.23 & 103.66 \\
& & $(3.27 \%)$ & $(100 \%)$ \\
$\mathrm{PB} \mathrm{H} \beta-4 / \mathrm{Ni}-\mathrm{V}$ & 286.59 & 288.95 & 103.73 \\
& $(16.66 \%)$ & $(6.60 \%)$ & $(100 \%)$ \\
PB-H $\beta-8 / \mathrm{Ni}-\mathrm{V}$ & 286.51 & 288.95 & 103.70 \\
& $(23.73 \%)$ & $(5.90 \%)$ & $(100 \%)$ \\
\hline PB H $\beta-16 / \mathrm{Ni}-\mathrm{V}$ & 286.45 & 289.08 & 103.61 \\
& $(17.99 \%)$ & $(1.88 \%)$ & $(100 \%)$ \\
\hline
\end{tabular}

Table S6. $\mathrm{H}_{2}$ desorbed from PB modified $\mathrm{H} \beta / \mathrm{Ni}-\mathrm{V}$ fresh catalysts.

\begin{tabular}{cccc}
\hline \multirow{2}{*}{ Samples } & \multicolumn{3}{c}{ Amount of desorbed $\mathrm{H}_{2}\left(\mu \mathrm{mol} \mathrm{H} / \mathrm{g}_{\text {cat }}\right)$} \\
\cline { 2 - 4 } $\mathrm{T} / \mathrm{T} / \mathrm{Ni}-\mathrm{V}$ & 62.4 & $\mathrm{~T}>400^{\circ} \mathrm{C}$ & Overall \\
$\mathrm{n}$ & 24.1 & 55.9 & 118.4 \\
$\mathrm{~PB}-\mathrm{H} \beta-4 / \mathrm{Ni}-\mathrm{V}$ & 30.6 & 220.4 & 244.5 \\
$\mathrm{~PB}-\mathrm{H} \beta-8 / \mathrm{Ni}-\mathrm{V}$ & & 292.2 & 322.9 \\
$\mathrm{~PB}-\mathrm{H} \beta-16 / \mathrm{Ni}-\mathrm{V}$ & 46.1 & 78.4 & 124.5 \\
\hline
\end{tabular}


Table S7. Components of crude bio-oil and the HDO products over $\mathrm{PB}-\mathrm{H} \beta-8 / \mathrm{Ni}-\mathrm{V}$ catalyst

\begin{tabular}{|c|c|c|}
\hline \multirow{2}{*}{ Components } & \multicolumn{2}{|c|}{ Mass Yield (\%) } \\
\hline & Crude Bio-oil & HDO run \\
\hline Guaiacols & 59.30 & 0 \\
\hline Creosol & 16.69 & 0 \\
\hline Guaiacol & 15.47 & 0 \\
\hline 4-ethylguaiacol & 11.45 & 0 \\
\hline trans-Isoeugenol & 7.71 & 0 \\
\hline 4-propylguaiacol & 3.03 & 0 \\
\hline Eugenol & 1.94 & 0 \\
\hline Vanillin & 1.72 & 0 \\
\hline Other guaiacols & 1.29 & 0 \\
\hline Phenols & 23.42 & 4.35 \\
\hline Phenol & 2.44 & 1.61 \\
\hline p-Cresol & 11.42 & 2.74 \\
\hline 3-ethyllphenol & 3.29 & 0 \\
\hline 3,4-dimethylphenol & 4.23 & 0 \\
\hline 4-propylphenol, & 1.02 & 0 \\
\hline 4-methylbenzenediol & 1.02 & 0 \\
\hline Aromatics & 0 & 44.93 \\
\hline Benzene & 0 & 19.73 \\
\hline Toluene & 0 & 15.68 \\
\hline Xylenes & 0 & 7.02 \\
\hline Mesitylene & 0 & 2.50 \\
\hline Other aromatics & 0 & 0 \\
\hline Ketones & 9.23 & 0 \\
\hline 3-methyl-1,2-Cyclopentanedione & 1.31 & 0 \\
\hline 2,3-dimethyl-2-Cyclopenten-1-one & 0.87 & 0 \\
\hline 3-ethyl-2-hydroxy--2-cyclopenten-1-one & 1.52 & 0 \\
\hline Other ketones & 5.53 & 0 \\
\hline Anisoles & 2.15 & 0 \\
\hline 3,4-Dimethoxytoluene & 0.93 & 0 \\
\hline 2,3-Dimethoxytoluene & 0.85 & 0 \\
\hline 1,2-dimethoxy-benzene & 0.37 & 0 \\
\hline Acids & 1.34 & 0 \\
\hline Acetic acid & 1.34 & 0 \\
\hline Others & 4.56 & 0 \\
\hline Furfural & 2.16 & 0 \\
\hline 5-methyl-2-Furancarboxaldehyde & 0.64 & 0 \\
\hline 2-Furanmethanol & 0.62 & 0 \\
\hline others & 1.13 & 0 \\
\hline
\end{tabular}




\begin{tabular}{lll}
\hline $\mathbf{C H}_{4}$ & 0 & 12.21 \\
\hline
\end{tabular}

Table S8. Characterization of the fresh and spent PB-Hß-8/Ni-V catalyst.

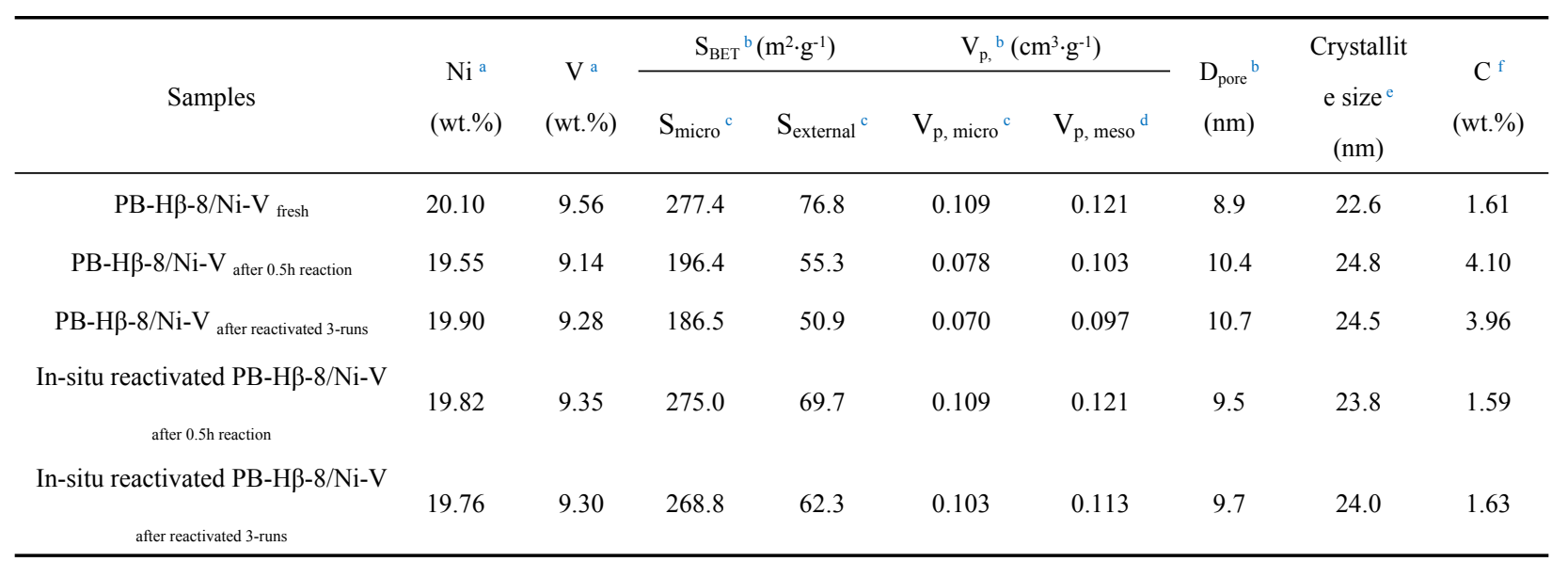
a. Determined by ICP-MS.
b. Determined by $\mathrm{N}_{2}$ isotherm adsorption-desorption.
c. Determined by using the t-Plot method.
d. Calculated by the difference between the total pore volume and the $\mathrm{Vp}$, micro
e. Calculated by the Scherrer equation using Ni (111) lattice planes.
f. Determined via TEM particle size distribution.
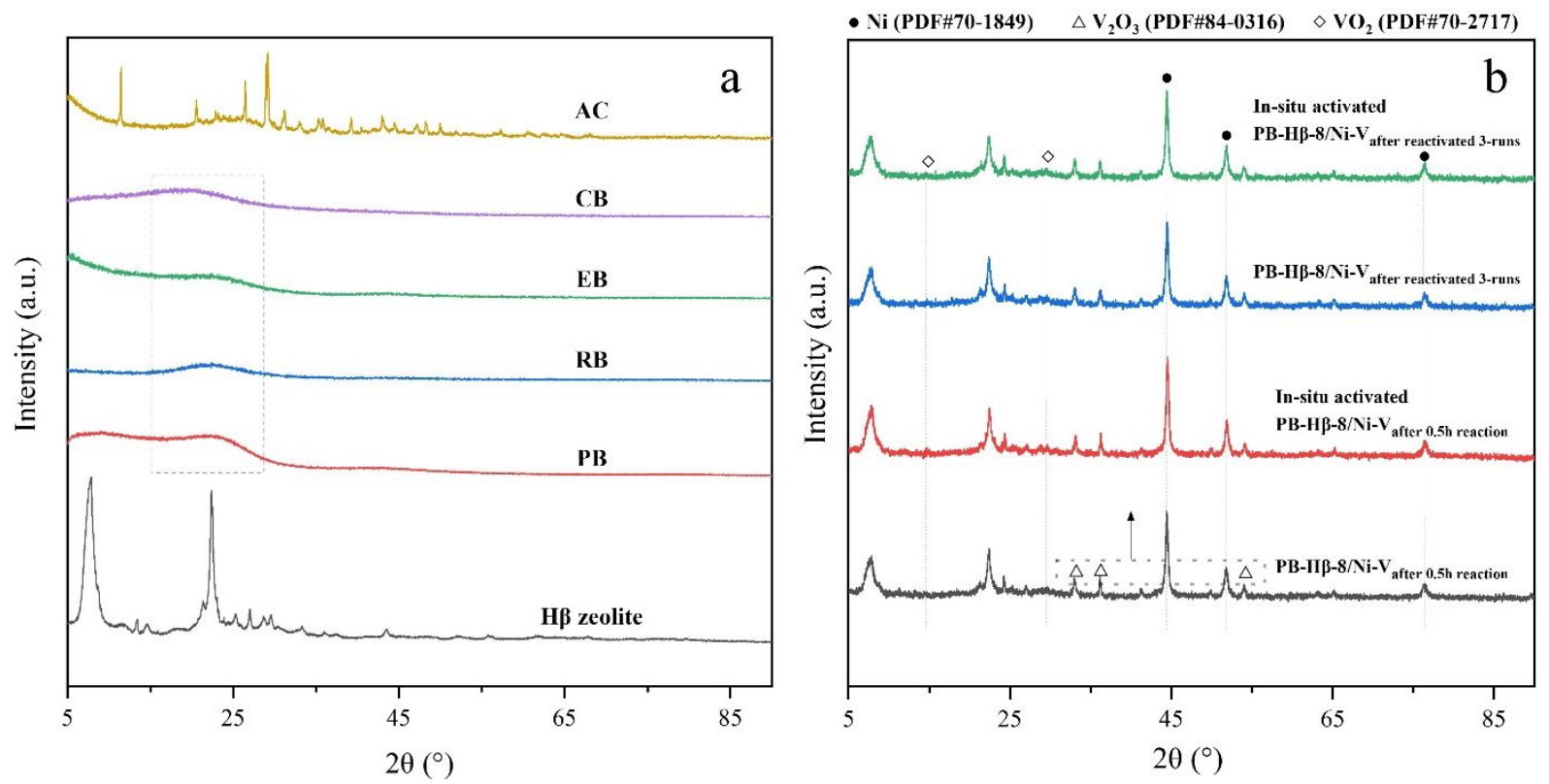

Figure S1. XRD patterns of the (a) different biochar and $\mathrm{H} \beta$ zeolite samples, (b) the spent and reactivated $\mathrm{PB}-\mathrm{H} \beta-8 / \mathrm{Ni}-\mathrm{V}$ catalysts. 

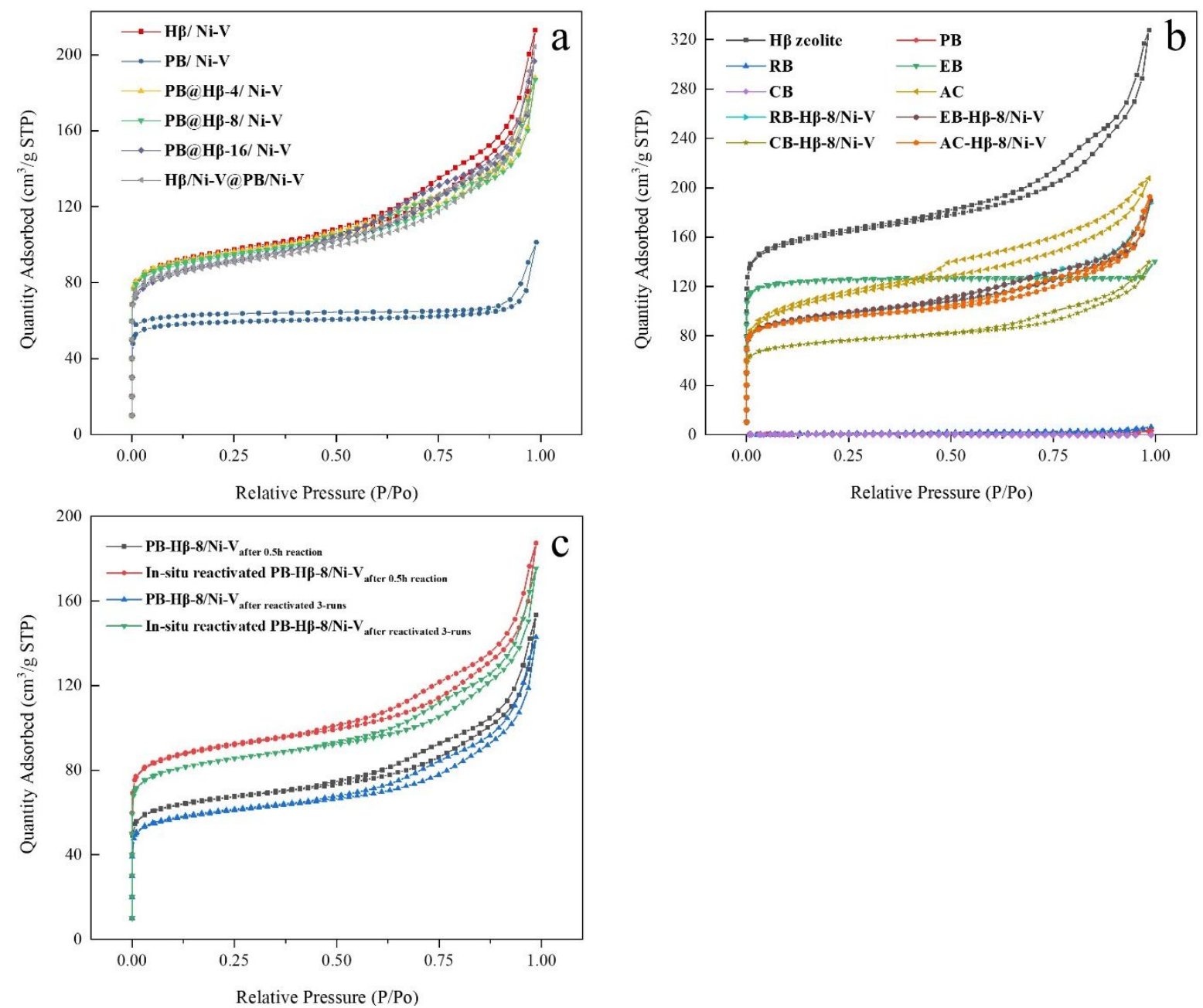

Figure S2. $\mathrm{N}_{2}$ adsorption-desorption isotherms of selected samples (a) $\mathrm{H} \beta / \mathrm{Ni}-\mathrm{V}, \mathrm{PB} / \mathrm{Ni}-\mathrm{V}$ and PB modified $\mathrm{H} \beta / \mathrm{Ni}-\mathrm{V}$ catalysts, (b) $\mathrm{H} \beta$, biochar and other biochar modified catalysts, (c) spent and reactivated $\mathrm{PB}-\mathrm{H} \beta-8 / \mathrm{Ni}-\mathrm{V}$ catalysts. 


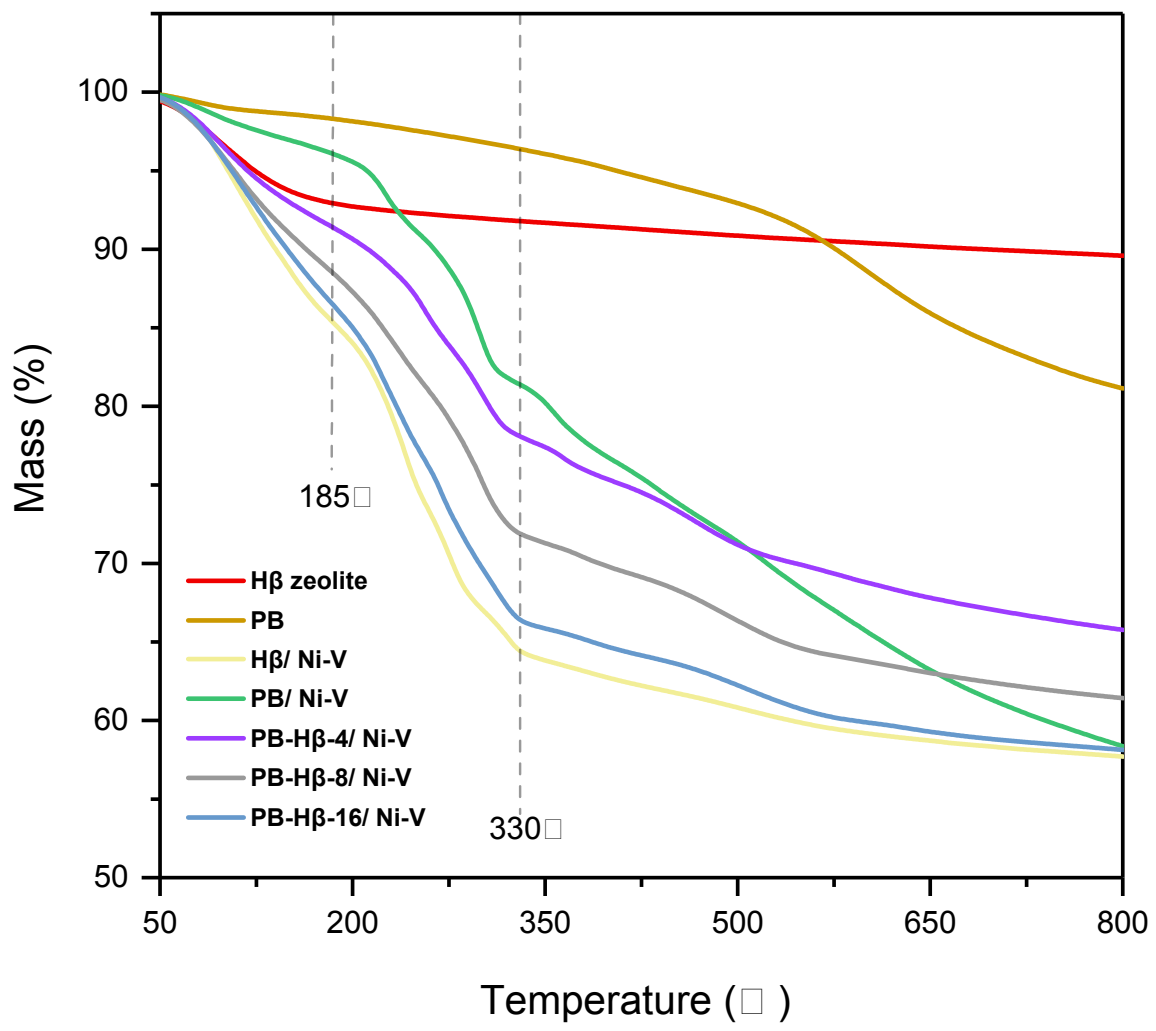

Figure S3. TG- $\mathrm{H}_{2}-\mathrm{TPR}$ profiles of the $\mathrm{H} \beta$ zeolite, $\mathrm{PB}$ and selected catalysts.

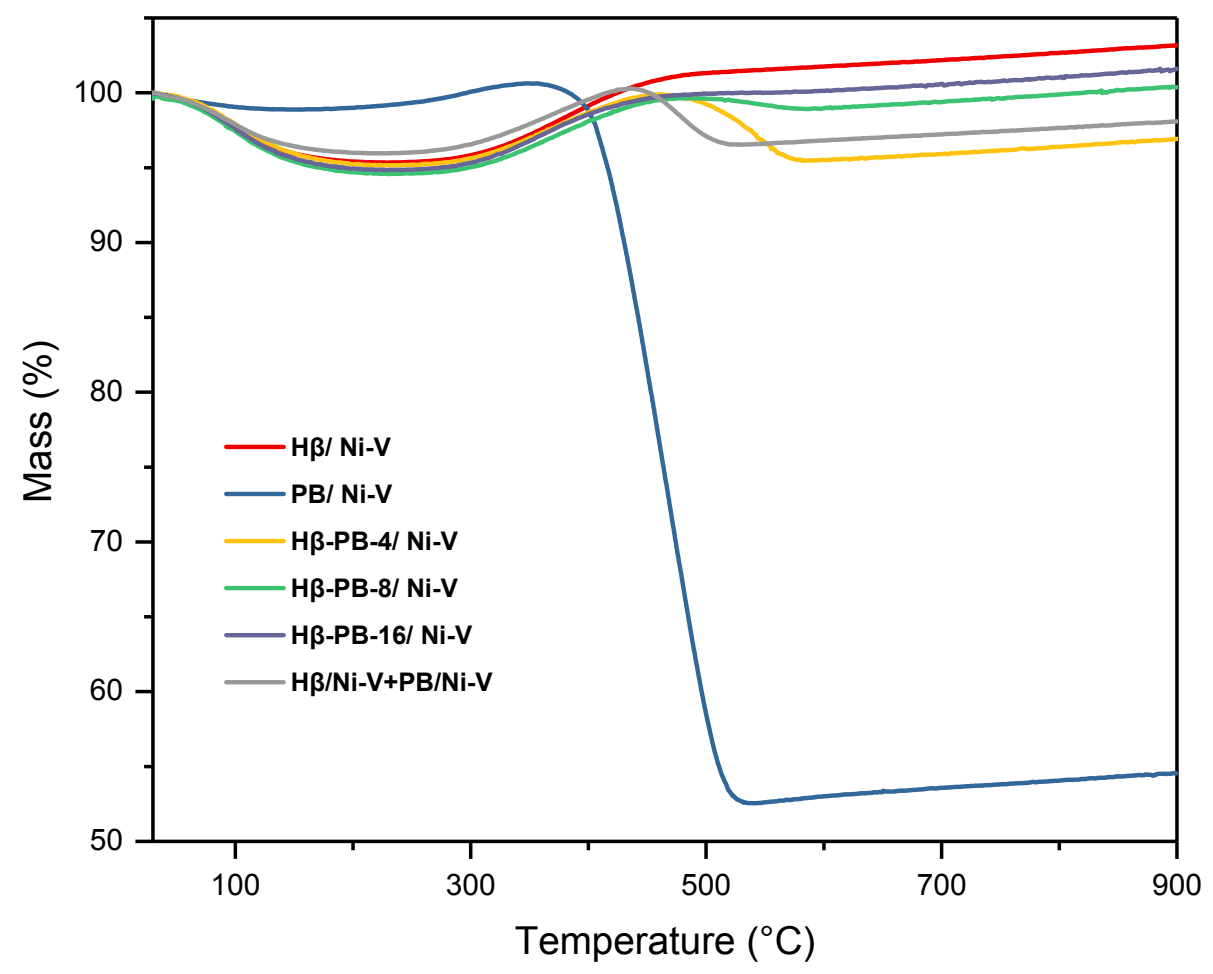

Figure S4. TG-TPO profiles of the fresh $\mathrm{H} \beta / \mathrm{Ni}-\mathrm{V}, \mathrm{PB} / \mathrm{Ni}-\mathrm{V}$ and $\mathrm{PB}$ modified $\mathrm{H} \beta / \mathrm{Ni}-\mathrm{V}$ catalysts. 
(a) C 1s core level spectra

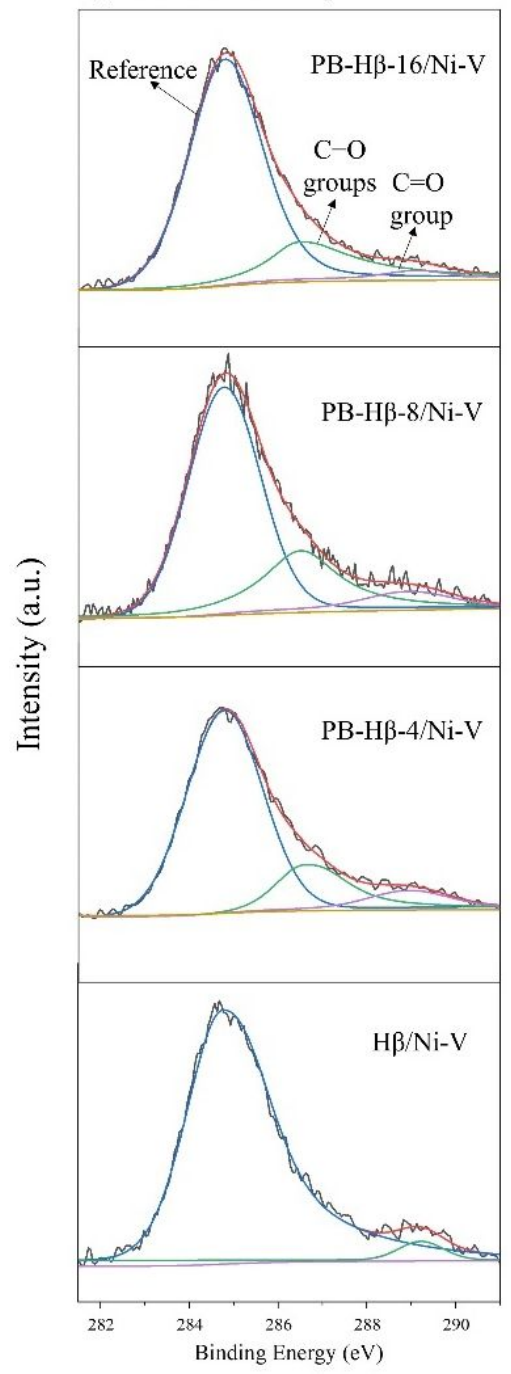

(b) Si 2p core level spectra

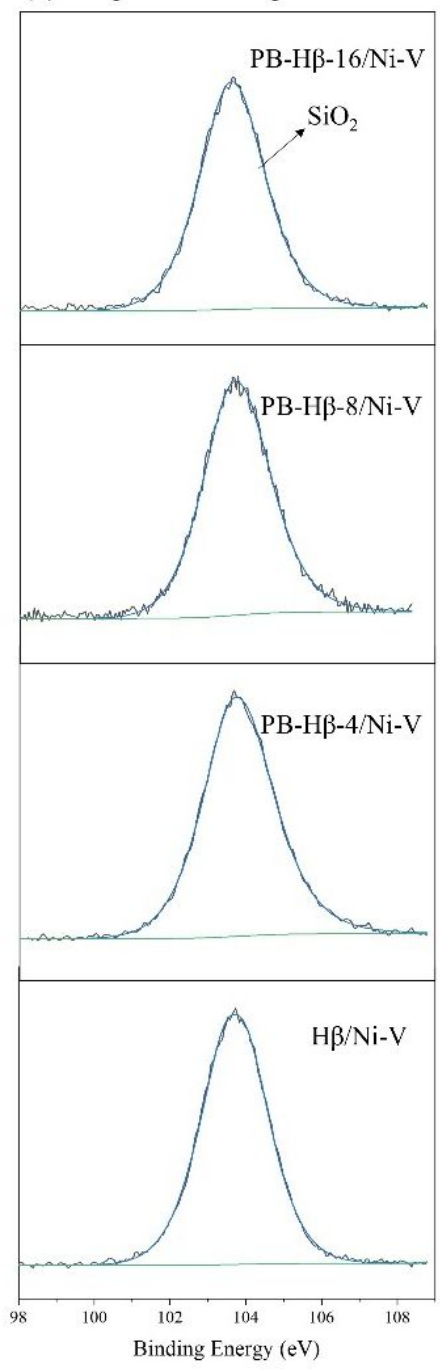

Figure S5. C 1s (a) and Si 2p (b) core-levels spectra of the selected catalysts. 


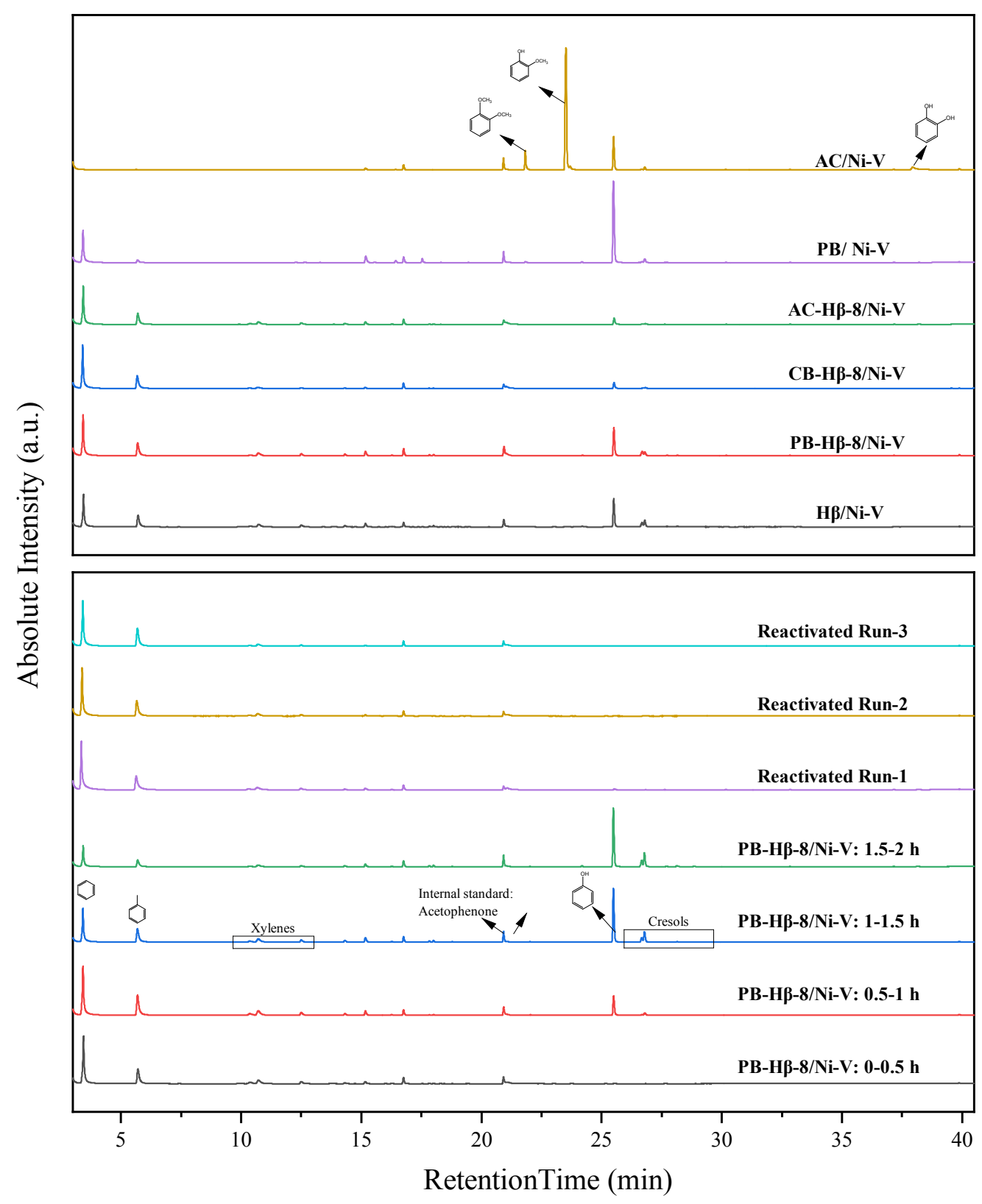

Figure S6. typical GC-MS graphs 


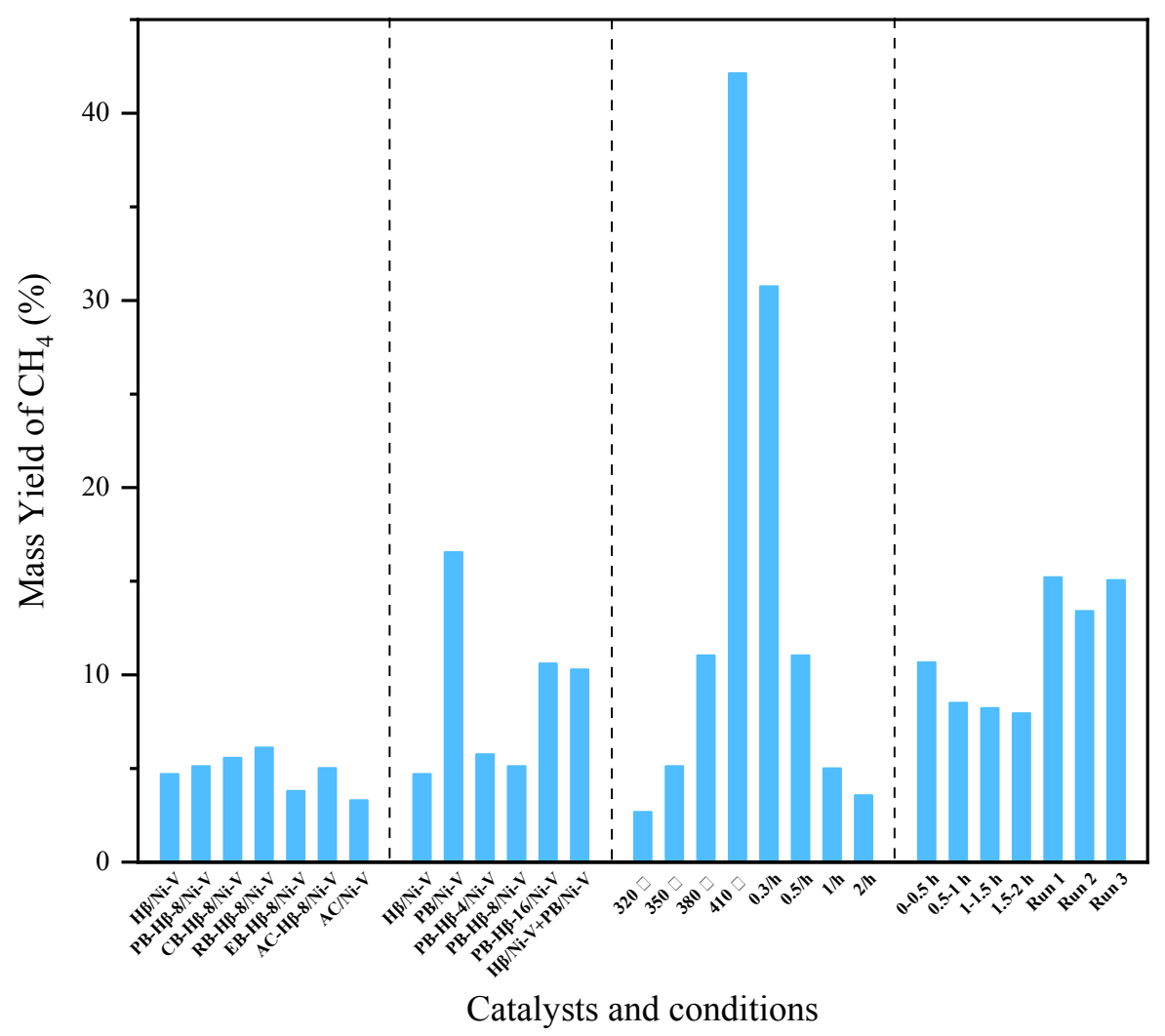

Figure S7. Mass yield of $\mathrm{CH} 4$ product via different catalysts and conditions

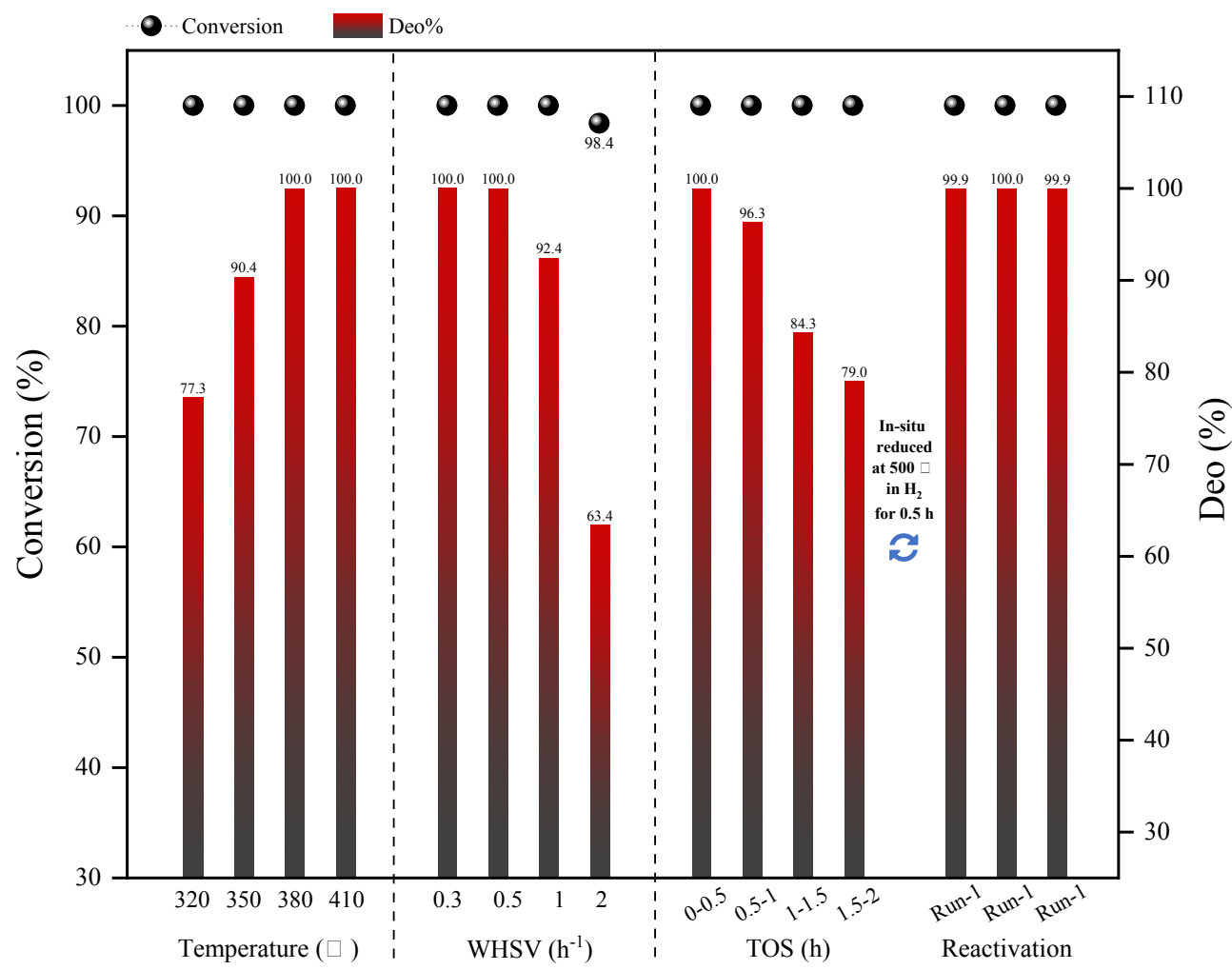

Figure S8. Conversion and Deo\% of $\mathrm{PB}-\mathrm{H} \beta-8 / \mathrm{Ni}-\mathrm{V}$ under different reaction temperature, 
WHSV, time conditions and reactivation test.

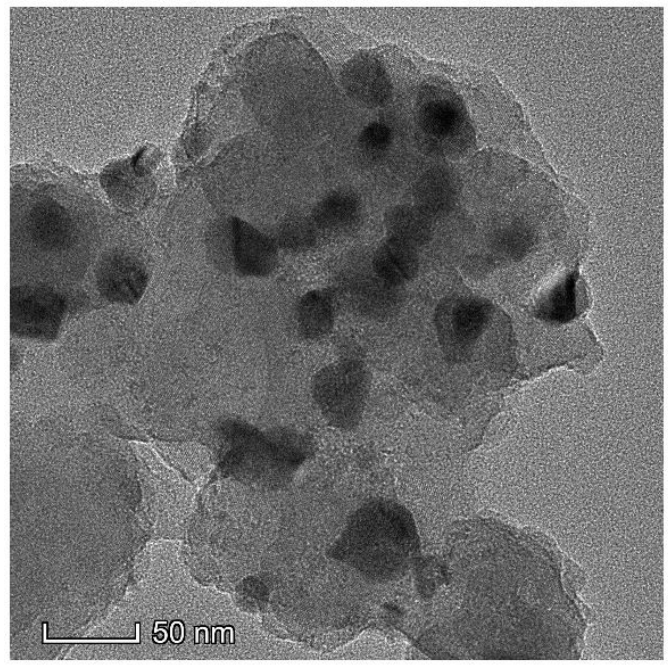

(a) $\mathrm{PB}-\mathrm{H} \beta-8 / \mathrm{Ni}-\mathrm{V}_{\text {after } 0.5 \mathrm{~h} \text { reaction }}$

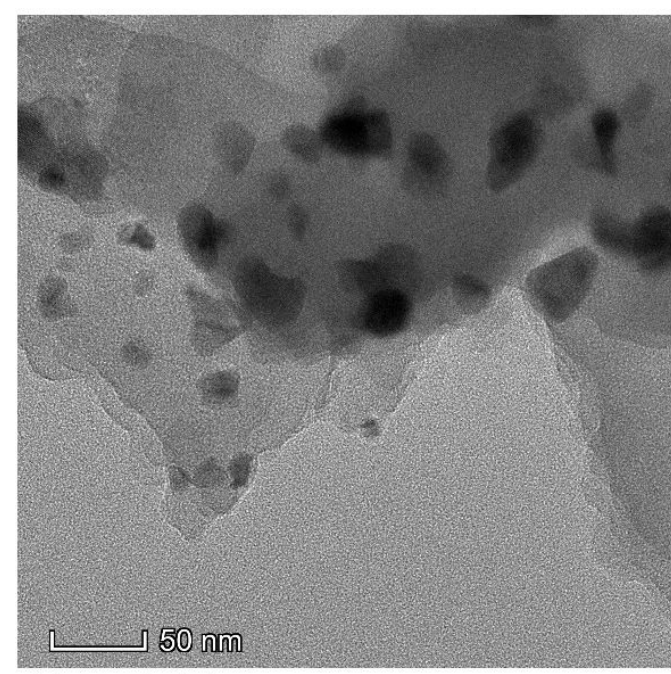

(b) $\mathrm{PB}-\mathrm{H} \beta-8 / \mathrm{Ni}-\mathrm{V}_{\text {after reactivated 3-runs }}$

Figure S9. TEM images of the spent $\mathrm{PB}$ modified $\mathrm{H} \beta / \mathrm{Ni}-\mathrm{V}$ catalysts (a) $\mathrm{PB}-\mathrm{H} \beta-8 / \mathrm{Ni}-\mathrm{V}_{\mathrm{after}} 0.5 \mathrm{~h}$ reaction (b) $\mathrm{PB}-\mathrm{H} \beta-8 / \mathrm{Ni}-\mathrm{V}_{\text {after reactivated 3-runs. }}$

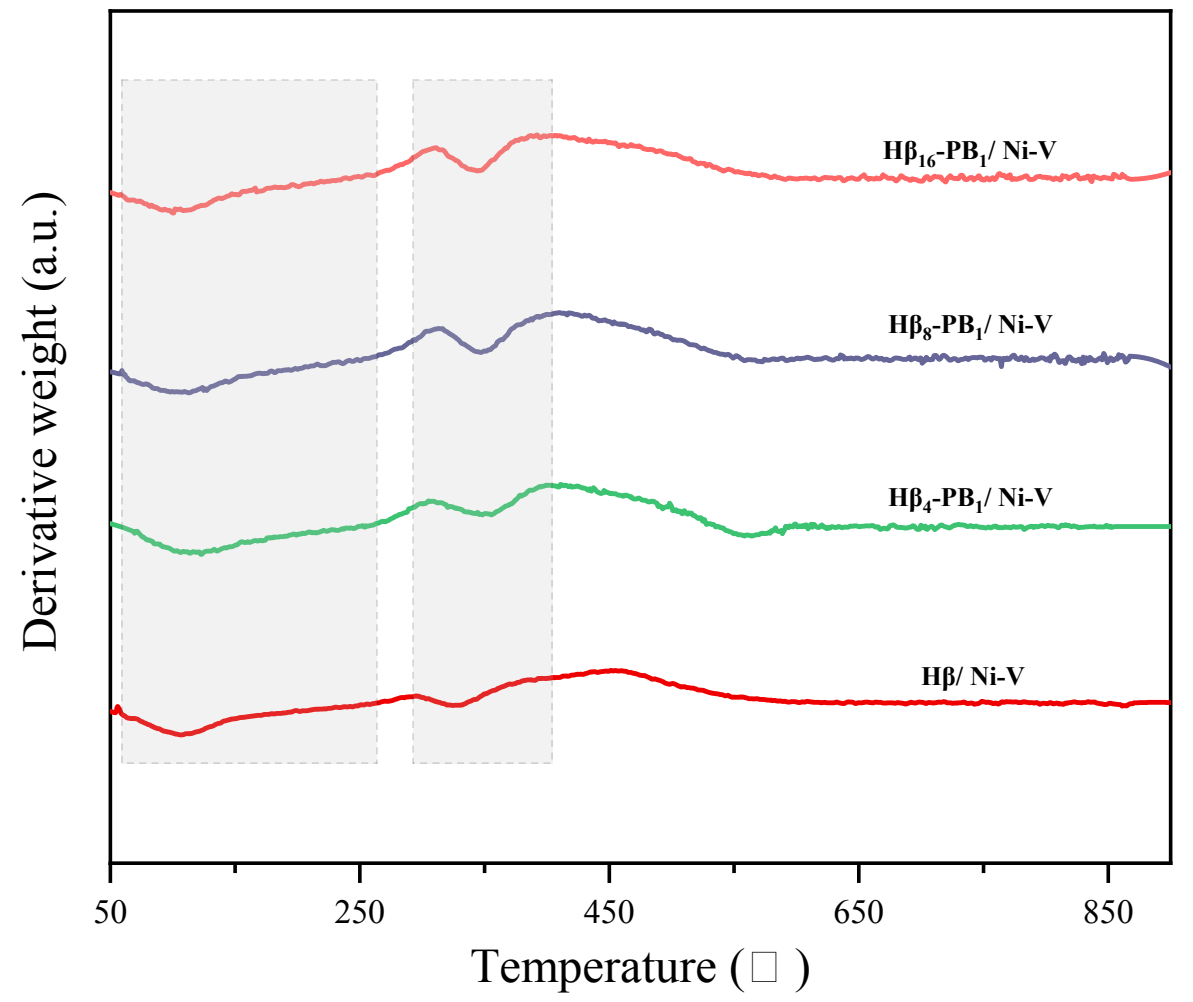

Figure S10. DTG-TPO profiles of spent $\mathrm{H} \beta / \mathrm{Ni}-\mathrm{V}$ and $\mathrm{PB}$ modified $\mathrm{H} \beta / \mathrm{Ni}-\mathrm{V}$ catalysts. 